\title{
Circulating osteocyte-derived exosomes contain miRNAs which are enriched in exosomes from MLO-Y4 cells
}

\author{
MARI SATO $^{1}$, TOMOHIDE SUZUKI ${ }^{2}$, MITSUOKI KAWANO ${ }^{3}$ and MASATO TAMURA ${ }^{1}$ \\ ${ }^{1}$ Department of Biochemistry and Molecular Biology, Graduate School of Dental Medicine, \\ Hokkaido University, Sapporo 060-8586; ${ }^{2}$ Hematology Division, Department of Medicine, \\ Kobe University Graduate School of Medicine, Kobe 650-0017; ${ }^{3}$ Department of Applied Life Sciences, \\ Niigata University of Pharmacy and Applied Life Sciences, Niigata 956-8603, Japan
}

Received September 1, 2016; Accepted October 27, 2016

DOI: $10.3892 /$ br.2016.824

\begin{abstract}
Signaling molecules produced by osteocytes have been proposed to serve as soluble factors that contribute to bone remodeling, as well as to homeostasis of other organs. However, to the best of our knowledge, there are currently no studies investigating the role of osteocyte-secreted exosomes. In the present study, ablation of osteocytes in mice [osteocyte-less (OL)] was used to examine the microRNA (miRNA) levels of plasma-circulating exosomes. In order to investigate the function of osteocyte-secreted exosomes, exosomes derived from MLO-Y4 cells were extracted and their miRNA expression levels were examined using miRNA array analysis and deep sequencing. Comparison of miRNA expression levels between plasma exosomes from OL mouse plasma and MLO-Y4-derived exosomes revealed that decreases in the number of miRNAs from exosomes circulating in the OL mouse plasma may be caused by a decrease in secretion of exosomes from osteocytes. These results suggest that osteocytes secrete exosomes containing characterized miRNAs and then circulate in the blood, and may thus transfer their components, including miRNAs, to recipient cells where they function as signaling molecules in other organs and/or tissues to regulate biological responses.
\end{abstract}

\section{Introduction}

Osteocytes are the most abundant cells in the bone, and are distributed in an interconnected network through which they sense and respond to systemic or local stimuli to regulate bone remodeling, exerting their effects via cell-cell interactions and soluble mediators $(1,2)$. It has been demonstrated that osteocytes release various soluble signaling molecules, such as receptor activator of nuclear factor- $\kappa \mathrm{B}$ ligand, insulin

Correspondence to: Professor Masato Tamura, Department of Biochemistry and Molecular Biology, Graduate School of Dental Medicine, Hokkaido University, North 13, West 7, Sapporo 060-8586, Japan

E-mail: mtamura@den.hokudai.ac.jp

Key words: osteocyte, exosome, microRNA, circulating exosome like growth factor-1, sclerostin, NO and ATP, which affect the osteoblastic and osteoclastic activities $(2,3)$. In addition to the regional mediators, molecules released from osteocytes, including fibroblast growth factor (FGF)23, dentin matrix protein (DMP)-1 and sclerostin have been detected in the serum of all healthy human subjects (3), indicating that these secreted proteins enter the circulation and are transported to distant organs. In fact, the main target organ of FGF23 is the kidney, where it suppresses phosphate reabsorption on tubular cells, implying that osteocytes regulate not only regional but also systemic processes by circulating soluble mediators $(3,4)$. Recently, Sato et al reported that ablation of osteocytes in mice leads to lymphopenia and loss of white adipose tissues (5). However, it remains unclear how osteocytes regulate distinct organs. Therefore, the regulatory mechanisms by which osteocytes function on distinct organs remains to be elucidated.

Extracellular vesicles are released by cells from multivesicular bodies (also known as exosomes) or from the plasma membrane (microvesicles) (6). Exosomes are released into the extracellular environment by a wide variety of cell types and are detected in the majority of biological fluids, including the plasma, breast milk, urine and saliva $(7,8)$. They contain lipids, proteins and nucleic acids consisting of DNA, as well as protein-coding and non-coding RNAs, including microRNAs (miRNAs or miRs) (6) Exosomes have been proposed to transfer these components to the recipient cell by binding to the cell surface through adhesion molecules, or by fusion and internalization by the recipient cell (9). Previous studies have indicated that nucleic acids, including miRNA and mRNA, are delivered to recipient cells in exosomes along with proteins and lipids, and that they trigger downstream signaling events in the recipient cells $(6,10)$.

miRNAs are small non-coding RNAs that are 22 nucleotides (nt) in length. To date, $>2,000$ miRNAs have been discovered in mammals, with certain of these expressed in a tissue-specific manner, which suggests that they serve specific roles in the determination of tissues during biological processes, such as cell differentiation, cell growth, apoptosis and carcinogenesis, and in various diseases $(11,12)$. There is accumulating evidence to suggest that regulation of cellular functions by miRNAs is a notable component of the regulatory system $(13,14)$. miRNAs are not only isolated inside cells, but are also transported in exosomes and/or microvesicles, apoptotic bodies and other 
microparticles. Export of intracellular miRNAs occurs partially through exosome-mediated mechanisms, and miRNAs in exosomes repress resident mRNA translation after entering recipient cells, thus regulating biological events, including angiogenesis, immune response and tumor cell invasion $(10,15,16)$.

The present study focuses on investigating the miRNA expression of osteocyte-derived exosomes. It was hypothesized that osteocytes may secrete exosomes containing characterized miRNAs; therefore the possibility that these exosomes may be present in the circulation was examined. Altered expression levels of miRNAs in plasma exosomes derived from osteocyte-less (OL) mice were observed, when compared with those in wild-type (WT) mice. According to miRNA profiling studies using exosomes prepared from mouse osteocyte MLO-Y4 cells and bone marrow stromal ST2 cells, osteocyte-derived exosomes were found to contain characterized miRNAs, and certain of those miRNAs were diminished in the exosomes obtained from the plasma of OL mice. These findings suggest that osteocytes in the bone tissue produce exosomes containing specific miRNAs, which circulate in the peripheral blood, from where they may be transferred to other organs.

\section{Materials and methods}

Mice and isolation of plasma. The study utilized a transgenic (Tg) mouse (DMP-1 DTR Tg) with targeted expression of diphtheria toxin receptor (DTR) under the promoter of DMP-1 (17). The DMP-1 DTR Tg mouse was backcrossed for more than nine generations onto a C57BL/6 background prior to use. The WT and Tg littermate mice (age, 15 weeks, body weight, $\sim 30 \mathrm{~g}$ ) were injected with a single dose of diphtheria toxin (DT; $20 \mu \mathrm{g} / \mathrm{kg}$, intraperitoneally; Sigma-Aldrich, St. Louis, MO, USA), and then plasma was harvested 3 weeks later (at 18 weeks). Mice were housed under a 12-h light/dark cycle at a temperature of $23-25^{\circ} \mathrm{C}$, humidity between $50-70 \%$ and were fed a normal diet. All animal experiments were approved by the Animal Care and Use Committee of Kobe University (Kobe, Japan), and were conducted according to the Kobe University Animal Experimentation Regulations.

Exosome isolation from plasma. Exosomes were isolated from the plasma using the Total Exosome Isolation kit (from plasma) (Invitrogen; Thermo Fisher Scientific, Inc., Waltham, MA, USA) according to the manufacturer's instructions. Briefly, 0.2 volume of the total exosome isolation reagent was added to the plasma and vortexed to mix. Samples were incubated overnight at $4^{\circ} \mathrm{C}$, and subsequently centrifuged at $15,000 \mathrm{xg}$ for $1 \mathrm{~h}$ at $4^{\circ} \mathrm{C}$. The supernatants were discarded and the exosome pellets were resuspended in $100 \mu \mathrm{l}$ phosphate-buffered saline (PBS).

Isolation of RNA. Total RNA containing the small RNA fraction was extracted using a mirVana miRNA isolation kit (Ambion; Thermo Fisher Scientific, Inc.) according to the manufacturer's instructions. This protocol effectively recovers both mRNA and miRNA. Subsequently, the concentration of the total RNA samples was evaluated using a NanoDrop ND-1000 spectrophotometer (Thermo Fisher Scientific, Inc.). The integrity of the total RNA samples was also analyzed using the Agilent 2100 Bioanalyzer Lab-on-a-Chip instrument system (Agilent Technologies, Santa Clara, CA, USA) with the
RNA 6000 Nano Chip (Applied Biosystems; Thermo Fisher Scientific, Inc.).

miRNA microarray and data analysis. Microarray analyses were performed using the 3D-Gene miRNA microarray platform (Toray Industries, Inc., Kamakura, Japan). Briefly, total RNA containing the small RNA fraction was labeled with a 3D-Gene miRNA labeling kit (Toray Industries, Inc.), and hybridized at $32^{\circ} \mathrm{C}$ for $16 \mathrm{~h}$ on the $3 \mathrm{D}-G e n e$ chip. The microarray was then scanned, and the obtained images were numerated using a 3D-Gene scanner 3000 (Toray Industries, Inc.). The microarray images obtained were analyzed using Genepix Pro 4.0 software (Molecular Devices, Sunnyvale, CA, USA). Differences in the total fluorescence intensity between arrays were adjusted by global normalization. The mean values for duplicate microarrays were calculated and used for comparison between the groups. When the difference in relative miRNA expression between the two groups was $>2.0$-fold, this was defined as a change in the expression. Subsequently, the expression level of each miRNA was globally normalized using the background-subtracted signal intensity of the entire miRNAs in each microarray. The mean values from duplicate microarrays were calculated and used for comparison between the groups.

Cell culture. The immortalized mouse osteocytic MLO-Y4 cell line was provided by Dr Lynda Bonewald (University of Missouri-Kansas City, School of Dentistry, Kansas City, MO, USA) (18). MLO-Y4 cells were cultured in $\alpha$-modified minimum essential medium (Sigma-Aldrich) containing 10\% fetal bovine serum (SAFC Bioscience, Inc., Lenexa, KS, USA) with $100 \mu \mathrm{g} / \mathrm{ml}$ kanamycin (Meiji, Ltd., Tokyo, Japan) and maintained in plates or flasks coated with $0.15 \mathrm{mg} / \mathrm{ml}$ rat tail collagen type I (BD Biosciences, Bedford, MA, USA) at $37^{\circ} \mathrm{C}$ in a humidified atmosphere of $5 \% \mathrm{CO}_{2}$ in air. In addition, the mouse stromal ST2 cell line was obtained from the RIKEN Cell Bank (Tsukuba, Japan), and cells were cultured as described previously (19).

Exosome isolation from cultured cells. At 3 days after reaching $100 \%$ confluence, culture medium was harvested and exosomes were isolated using the Total Exosome Isolation Reagent (from cell culture media) (Invitrogen; Thermo Fisher Scientific, Inc.) according to the manufacturer's instructions. Briefly, in order to remove cellular debris, the cell culture medium was centrifuged at 2,000 $\mathrm{x}$ g for $20 \mathrm{~min}$, and the supernatant was transferred to a new microfuge tube. Subsequently, 0.5 volumes of reagent were added to the supernatant and vortexed to mix. Samples were incubated overnight at $4^{\circ} \mathrm{C}$, and then centrifuged at $15,000 \mathrm{xg}$ for $1 \mathrm{~h}$ at $4^{\circ} \mathrm{C}$. The supernatants were discarded and each pellet was resuspended in $100 \mu \mathrm{l}$ PBS.

Small RNA library preparation and deep sequencing. RNA $(1 \mu \mathrm{g})$ was converted into a cDNA library using a TruSeq Small RNA Library Preparation kit (cat. no. RS-200-0012; Illumina, Inc., San Diego, CA, USA) according the manufacturer's protocol. Briefly, 5' and 3' adaptors were ligated, followed by reverse transcription using SuperScript II reverse transcriptase (Invitrogen; cat. no. 18064014) and library enrichment by PCR amplification. The PCR conditions were as follows: Initial denaturation at $98^{\circ} \mathrm{C}$ for $30 \mathrm{sec}$, followed by 13 cycles of $98^{\circ} \mathrm{C}$ for $10 \mathrm{sec}, 60^{\circ} \mathrm{C}$ for $30 \mathrm{sec}, 72^{\circ} \mathrm{C}$ for $15 \mathrm{sec}$, and a final extension 
step at $72^{\circ} \mathrm{C}$ for $10 \mathrm{~min}$. Following enrichment, the cDNA library was purified and run on a $6 \%$ polyacrylamide gel, and then the appropriate band (18-30 nt in size) was excised and eluted. The purified cDNA library was run on a HiSeq 2500 ultra-high-throughput sequencing system (Illumina, Inc.) with read length of $101 \mathrm{nt}$, and the resulting reads were analyzed.

Analysis of small RNA sequence data. In order to perform real-time image analysis and base calling, the HiSeq Control Software (Illumina, Inc.) were used on HiSeq 2500 (Illumina, Inc.). Raw readings, passed through a chastity filter, were initially extracted from the FASTQ files. and the Cutadapt application (https://cutadapt.readthedocs.io/en/stable/) was used to remove the adaptor sequences from each sequence read. Any sequences with $<10 \mathrm{nt}$ after trimming were not used. The prepared sequences were aligned using the BLAST+ tool (version 2.2.29+) downloaded via FTP from ftp://ftp.ncbi.nlm. nih.gov/blast/db/ against mouse miRNA sequences miRBase (Release 21; http://www.mirbase.org/).

\section{Results}

Altered miRNA expression levels in exosomes derived from the plasma of $O L$ mice. To elucidate whether exosomes from osteocytes circulate in the peripheral blood, a Tg mouse model with targeted expression of DTR under the control of the DMP-1 promoter was used (17). WT and Tg littermate mice (15-week-old) were injected with DT. After 3 weeks, the DT-injected mice showed a comparable lacuno-canalicular interstitial fluid space to that of WT mice; however, there was a marked reduction in the osteocyte network, and thuse these mice were denoted as OL $(5,20)$. In the initial attempt to identify differentially expressed miRNAs between the plasma exosomes of OL and WT mice, exosomes were isolated from plasma samples, and then total RNA was extracted from the exosome preparations. The quality and quantity of these RNA isolates were determined using a bioanalyzer. In exosomal RNA derived from the OL and WT plasma samples, bioanalyzer profiles indicated that these RNAs lack detectable quantities of $18 \mathrm{~S}$ and $28 \mathrm{~S}$ ribosomal RNA, indicating that significant amounts of RNA are present (data not shown).

In order to investigate the miRNA content of these exosomes, the expression of miRNA was profiled using a 3D-Gene miRNA microarray platform that can detect $\sim 1,300$ known miRNAs. The normalized data were globally normalized per array, such that the median of the signal intensity was adjusted to 25 . The number of expressed (detected) miRNAs present at significant levels (global normalization value of $>100$ ) was determined. Among the expressed miRNAs in the plasma exosomes, the expression level of 30 miRNAs was downregulated $\left(\log _{2}\right.$ value; fold-change $<0.5$ ) and that of another 30 miRNAs was upregulated $\left(\log _{2}\right.$ value $\left.>1.5\right)$ in OL mice compared with the expression in WT mice (Table I). Among the downregulated miRNAs in OL mouse plasma, the relative expression levels of miR-3473a, miR-3473b, miR-3473e, miR-5128, miR-6244, miR-6239, miR-5132-5p, miR-705, miR-208a-5p miR-3104-5p, miR-1224-5p and miR-5621-5p were -1.0 or lower ( $\log _{2}$ value) compared with the WT mice (Table I). These results indicated that the expression levels of certain miRNAs were altered in exosomes derived from the plasma of OL mice.
Table I. Alterations in the plasma exosomal miRNA expression levels from OL mice.

\begin{tabular}{|c|c|}
\hline miR ID & Relative miRNA expression ${ }^{a}$ \\
\hline mmu-miR-3473a & -1.90 \\
\hline mmu-miR-3473b & -1.52 \\
\hline mmu-miR-3473e & -1.51 \\
\hline mmu-miR-5128 & -1.43 \\
\hline mmu-miR-6244 & -1.27 \\
\hline mmu-miR-6239 & -1.24 \\
\hline mmu-miR-5132-5p & -1.13 \\
\hline mmu-miR-705 & -1.10 \\
\hline mmu-miR-208a-5p & -1.09 \\
\hline mmu-miR-3104-5p & -1.07 \\
\hline mmu-miR-1224-5p & -1.05 \\
\hline mmu-miR-5621-5p & -1.03 \\
\hline mmu-miR-328-5p & -0.87 \\
\hline mmu-miR-5130 & -0.87 \\
\hline mmu-miR-652-5p & -0.84 \\
\hline mmu-miR-5112 & -0.76 \\
\hline mmu-miR-149-3p & -0.73 \\
\hline mmu-miR-346-3p & -0.71 \\
\hline mmu-miR-5109 & -0.69 \\
\hline mmu-miR-3102-5p & -0.68 \\
\hline mmu-miR-6370 & -0.64 \\
\hline mmu-miR-6240 & -0.63 \\
\hline mmu-miR-486-3p & -0.62 \\
\hline mmu-miR-326-5p & -0.61 \\
\hline mmu-miR-6385 & -0.60 \\
\hline mmu-miR-5105 & -0.59 \\
\hline mmu-miR-615-5p & -0.59 \\
\hline mmu-miR-6405 & -0.58 \\
\hline mmu-miR-5126 & -0.56 \\
\hline mmu-miR-5119 & -0.52 \\
\hline
\end{tabular}

B, Upregulated miRNAs

$\operatorname{miR}$ ID

Relative miRNA expression ${ }^{\mathrm{a}}$

mmu-miR-122-5p

3.65

mmu-miR-133b-3p

2.67

mmu-miR-133a-3p

2.51

mmu-miR-25-3p

2.31

mmu-miR-92b-3p

2.19

mmu-miR-19a-3p

2.19

mmu-miR-92a-3p

2.08

mmu-miR-144-3p

2.06

mmu-miR-15b-5p

2.03

mmu-miR-29c-3p

2.00

mmu-miR-486-5p

1.99

mmu-miR-93-5p

1.93

mmu-miR-15a-5p

1.80

mmu-miR-221-3p 
Table I. Continued.

B, Upregulated miRNAs

\begin{tabular}{lc}
\hline miR ID & Relative miRNA expression ${ }^{\mathrm{a}}$ \\
\hline mmu-miR-192-5p & 1.76 \\
mmu-miR-19b-3p & 1.74 \\
mmu-let-7j & 1.71 \\
mmu-miR-451a & 1.71 \\
mmu-let-7i-5p & 1.70 \\
mmu-miR-103-3p & 1.69 \\
mmu-miR-107-3p & 1.66 \\
mmu-miR-18a-5p & 1.63 \\
mmu-miR-195a-5p & 1.60 \\
mmu-miR-484 & 1.59 \\
mmu-miR-16-5p & 1.58 \\
mmu-miR-18b-5p & 1.58 \\
mmu-miR-30a-5p & 1.54 \\
mmu-miR-425-5p & 1.52 \\
mmu-miR-301a-3p & 1.51 \\
mmu-miR-29a-3p & 1.51
\end{tabular}

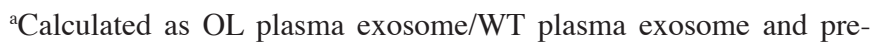
sented as a $\log _{2}$ value. The expression levels of 30 miRNAs were lower $\left(\log _{2}\right.$ value $\left.<0.5\right)$ and those of 30 miRNAs were higher $\left(\log _{2}\right.$ value $>1.5$ ) in plasma exosomes from OL mice compared with WT mice. OL, osteocyte-less; miRNA or miR, microRNA.

Table II. Enriched expression of miRNAs in exosomes from MLO-Y4 cells compared with ST2 cells

\begin{tabular}{lll}
\hline & \multicolumn{2}{c}{ Relative miRNA expression ${ }^{\mathrm{a}}$} \\
\cline { 2 - 3 } miR ID & $\begin{array}{c}\text { MLO-Y4/ST2 } \\
\text { exosomes }\end{array}$ & $\begin{array}{c}\text { MLO-Y4 exosomes/ } \\
\text { MLO-Y4 cells }\end{array}$ \\
\hline mmu-miR-3473e & 5.66 & 3.77 \\
mmu-miR-1224-5p & 5.34 & 5.80 \\
mmu-miR-1940 & 5.07 & 5.20 \\
mmu-miR-5128 & 4.8 & 4.51 \\
mmu-miR-3473b & 4.64 & 3.79 \\
mmu-miR-3473a & 4.58 & 3.02 \\
mmu-miR-29a-3p & 4.45 & 0.67 \\
mmu-miR-1964-5p & 4.29 & 6.06 \\
mmu-miR-140-3p & 4.06 & 1.79 \\
mmu-miR-6240 & 4.03 & 3.24 \\
mmu-miR-5099 & 3.92 & 3.06 \\
mmu-miR-5129-5p & 3.82 & n.d. \\
mmu-miR-23a-3p & 3.77 & 1.43 \\
mmu-miR-690 & 3.65 & 0.53 \\
mmu-miR-23b-3p & 3.48 & 1.63 \\
mmu-miR-365-1-5p & 3.46 & n.d. \\
mmu-miR-1930-3p & 3.36 & n.d. \\
mmu-miR-130a-3p & 3.28 & 1.71 \\
mmu-miR-491-5p & 3.17 & n.d. \\
mm & &
\end{tabular}

Table II. Continued.

\begin{tabular}{|c|c|c|}
\hline \multirow[b]{2}{*}{$\operatorname{miR} I D$} & \multicolumn{2}{|c|}{ Relative miRNA expression } \\
\hline & $\begin{array}{l}\text { MLO-Y4/ST2 } \\
\text { exosomes }\end{array}$ & $\begin{array}{c}\text { MLO-Y4 exosomes/ } \\
\text { MLO-Y4 cells }\end{array}$ \\
\hline mmu-miR-193b-5p & 3.15 & n.d. \\
\hline mmu-miR-6236 & 3.11 & 3.35 \\
\hline mmu-miR-214-3p & 3.08 & 2.47 \\
\hline mmu-miR-5121 & 3.06 & n.d. \\
\hline mmu-miR-5627-5p & 3.05 & 1.33 \\
\hline mmu-miR-1247-3p & 2.95 & 3.16 \\
\hline mmu-miR-16-5p & 2.96 & -0.78 \\
\hline mmu-miR-705 & 2.94 & 4.05 \\
\hline mmu-miR-22-3p & 2.91 & 3.35 \\
\hline mmu-miR-92a-3p & 2.91 & 2.27 \\
\hline mmu-miR-27a-3p & 2.91 & 0.30 \\
\hline mmu-miR-1965 & 2.85 & 2.54 \\
\hline mmu-miR-24-3p & 2.85 & 1.82 \\
\hline mmu-miR-3072-5p & 2.83 & 3.50 \\
\hline mmu-miR-221-3p & 2.84 & 1.43 \\
\hline mmu-miR-3090-5p & 2.83 & -0.10 \\
\hline mmu-miR-125b-5p & 2.84 & -0.27 \\
\hline mmu-miR-671-5p & 2.82 & 2.81 \\
\hline mmu-miR-6244 & 2.8 & 2.20 \\
\hline mmu-miR-5620-5p & 2.8 & n.d. \\
\hline mmu-miR-6349 & 2.72 & n.d. \\
\hline mmu-miR-3104-5p & 2.71 & 3.19 \\
\hline mmu-miR-326-5p & 2.7 & 2.47 \\
\hline mmu-miR-5131 & 2.67 & 2.45 \\
\hline mmu-miR-125a-3p & 2.68 & 0.73 \\
\hline mmu-miR-762 & 2.64 & 2.10 \\
\hline mmu-miR-2136 & 2.65 & n.d. \\
\hline mmu-miR-744-5p & 2.59 & 2.77 \\
\hline mmu-miR-17-5p & 2.58 & -0.88 \\
\hline mmu-miR-680 & 2.57 & 3.66 \\
\hline mmu-miR-5622-3p & 2.55 & 1.62 \\
\hline mmu-miR-6406 & 2.54 & n.d. \\
\hline mmu-miR-19b-3p & 2.51 & 0.49 \\
\hline mmu-miR-665-3p & 2.51 & n.d. \\
\hline
\end{tabular}

Values are presented as $\log _{2}$ values. The expression of 53 miRNAs was higher $\left(\log _{2}\right.$ value $\left.>2.5\right)$ in MLO-Y4 exosomes compared with ST2 exosomes. miRNA or miR, microRNA; n.d., not detected.

MLO-Y4 cells released from exosomes contain miRNAs. In the present study, the MLO-Y4 osteocyte-like cell line was used as the osteocyte model for several reasons: i) These cells are isolated from weight-bearing bone and are a well-characterized osteocyte model that has been intensively studied since 1997 (18); and ii) MLO-Y4 cells express early osteocyte markers in vivo, such as E11, CD44 and low levels of the mature osteocyte marker sclerostin (3). By contrast, the ST2 cell line is a bone marrow-derived stromal cell line (21). ST2 cells cultured with ascorbic acid exhibit characteristics typical 
Table III. Downregulated miRNAs in exosomes from osteocyte-less mouse plasma and MLO-Y4 cellular and exosomal enriched miRNA.

\begin{tabular}{|c|c|c|c|}
\hline \multirow[b]{2}{*}{ miR ID } & \multicolumn{3}{|c|}{ Relative miRNA expression } \\
\hline & $\begin{array}{c}\text { OL plasma } \\
\text { exosome/WT plasma exosome }\end{array}$ & $\begin{array}{c}\text { MLO-Y4 } \\
\text { exosomes/ST2 exosomes }\end{array}$ & $\begin{array}{c}\text { MLO-Y4 } \\
\text { exosomes/MLO-Y4 cells }\end{array}$ \\
\hline mmu-miR-3473a & -1.90 & 4.58 & 3.02 \\
\hline mmu-miR-3473b & -1.52 & 4.64 & 3.79 \\
\hline mmu-miR-3473e & -1.51 & 5.66 & 3.77 \\
\hline mmu-miR-5128 & -1.43 & 4.8 & 4.51 \\
\hline mmu-miR-6244 & -1.27 & 2.8 & 2.20 \\
\hline mmu-miR-6239 & -1.24 & 2.15 & n.d. \\
\hline mmu-miR-5132-5p & -1.13 & 2.42 & 2.63 \\
\hline mmu-miR-705 & -1.10 & 2.94 & 4.05 \\
\hline mmu-miR-208a-5p & -1.09 & n.d. & n.d. \\
\hline mmu-miR-3104-5p & -1.07 & 2.71 & 3.19 \\
\hline mmu-miR-1224-5p & -1.05 & 5.34 & 5.80 \\
\hline mmu-miR-5621-5p & -1.03 & 2.26 & 2.29 \\
\hline mmu-miR-328-5p & -0.87 & 2.25 & 3.97 \\
\hline mmu-miR-5130 & -0.87 & 1.5 & 3.58 \\
\hline mmu-miR-652-5p & -0.84 & n.d. & n.d. \\
\hline mmu-miR-5112 & -0.76 & n.d. & n.d. \\
\hline mmu-miR-149-3p & -0.73 & 2.13 & 3.62 \\
\hline mmu-miR-346-3p & -0.71 & 1.72 & 2.51 \\
\hline mmu-miR-5109 & -0.69 & 2.49 & 1.99 \\
\hline mmu-miR-3102-5p.2-5p & -0.68 & 1.18 & 2.66 \\
\hline mmu-miR-6370 & -0.64 & 2.25 & n.d. \\
\hline mmu-miR-6240 & -0.63 & 4.03 & 3.24 \\
\hline mmu-miR-486-3p & -0.62 & 2.2 & 0.94 \\
\hline mmu-miR-326-5p & -0.61 & 2.7 & 2.47 \\
\hline mmu-miR-6385 & -0.60 & n.d. & n.d. \\
\hline mmu-miR-5105 & -0.59 & 2.24 & 3.26 \\
\hline mmu-miR-615-5p & -0.59 & 1.12 & n.d. \\
\hline mmu-miR-6405 & -0.58 & n.d. & n.d. \\
\hline mmu-miR-5126 & -0.56 & 1.76 & 2.34 \\
\hline mmu-miR-5119 & -0.52 & n.d. & n.d. \\
\hline
\end{tabular}

Values are presented as $\log _{2}$ values. miRNA or miR, microRNA; n.d., not detected.

of osteoblasts, including the formation of mineralized nodules, indicating that ST2 cells are pre-osteoblastic stromal cells (22). Therefore, ST2 cells were used as control non-osteocytic cells in the present study.

In order to identify differentially-expressed miRNAs in exosomes obtained from MLO-Y4 and ST2 cells, exosomes were isolated from culture supernatants, and the RNA was isolated. Bioanalyzer profiles indicated that these exosomal RNAs lacked detectable amounts of ribosomal RNA, whereas total cellular RNAs contained ribosomal 18s and 28s RNAs and mRNAs as major components, indicating that significant amounts of small RNAs were present (data not shown). Using miRNA microarray analysis, the number of miRNAs expressed at significant levels in the exosomes prepared from MLO-Y4 and ST2 cells was detected. Analysis of these miRNA profiles revealed that
53 miRNAs were enriched at significantly higher levels $\left(\log _{2}\right.$ value $>2.5$ ) in MLO-Y4 cells compared with their levels in ST2 cells (Table II). Furthermore, the miRNA expression profile between the exosomal and cellular fractions of MLO-Y4 cells was compared (Table II). Taken together, these data demonstrated that characterized miRNAs were present in the MLO-Y4 exosomes, and $71.7 \%(38 / 53)$ of these miRNAs were enriched in the exosomes compared with their expression in host cells.

Comparison of miRNA expression between plasma exosomes from OL mice and MLO-Y4-derived exosomes. To determine whether exosomes derived from osteocytes are circulating in the blood, the miRNA expression levels of exosomes were analyzed and compared between OL mouse plasma and MLO-Y4 cells. Downregulated and upregulated miRNAs in 
Table IV. Upregulated miRNAs in exosomes from osteocyte-less mouse plasma and MLO-Y4 cellular and exosomal miRNA.

\begin{tabular}{|c|c|c|c|}
\hline \multirow[b]{2}{*}{ miR ID } & \multicolumn{3}{|c|}{ Relative miRNA expression } \\
\hline & $\begin{array}{c}\text { OL plasma } \\
\text { exosome/WT plasma exosome }\end{array}$ & $\begin{array}{c}\text { MLO-Y4 } \\
\text { exosomes/ST2 exosomes }\end{array}$ & $\begin{array}{c}\text { MLO-Y4 } \\
\text { exosomes/MLO-Y4 cells }\end{array}$ \\
\hline mmu-miR-133b-3p & 2.67 & n.d. & n.d. \\
\hline mmu-miR-133a-3p & 2.51 & n.d. & n.d. \\
\hline mmu-miR-25-3p & 2.31 & n.d. & n.d. \\
\hline mmu-miR-92b-3p & 2.19 & n.d. & n.d. \\
\hline mmu-miR-19a-3p & 2.19 & n.d. & n.d. \\
\hline mmu-miR-92a-3p & 2.08 & 2.91 & 2.27 \\
\hline mmu-miR-144-3p & 2.06 & n.d. & n.d. \\
\hline mmu-miR-15b-5p & 2.03 & n.d. & n.d. \\
\hline mmu-miR-29c-3p & 2.00 & n.d. & n.d. \\
\hline mmu-miR-486-5p & 1.99 & n.d. & n.d. \\
\hline mmu-miR-93-5p & 1.93 & n.d. & n.d. \\
\hline mmu-miR-15a-5p & 1.80 & n.d. & n.d. \\
\hline mmu-miR-221-3p & 1.77 & 2.84 & 1.43 \\
\hline mmu-miR-192-5p & 1.76 & n.d. & n.d. \\
\hline mmu-miR-19b-3p & 1.74 & 2.51 & 0.49 \\
\hline mmu-let-7j & 1.71 & n.d. & n.d. \\
\hline mmu-miR-451a & 1.71 & 1.04 & n.d. \\
\hline mmu-let-7i-5p & 1.70 & n.d. & n.d. \\
\hline mmu-miR-103-3p & 1.69 & n.d. & n.d. \\
\hline mmu-miR-107-3p & 1.66 & n.d. & n.d. \\
\hline mmu-miR-18a-5p & 1.63 & n.d. & n.d. \\
\hline mmu-miR-195a-5p & 1.60 & n.d. & n.d. \\
\hline mmu-miR-484 & 1.59 & 2.3 & 2.66 \\
\hline mmu-miR-16-5p & 1.58 & 2.96 & -0.78 \\
\hline mmu-miR-18b-5p & 1.58 & n.d. & n.d. \\
\hline mmu-miR-30a-5p & 1.54 & n.d. & n.d. \\
\hline mmu-miR-425-5p & 1.52 & n.d. & n.d. \\
\hline mmu-miR-301a-3p & 1.51 & n.d. & n.d. \\
\hline mmu-miR-29a-3p & 1.51 & 4.45 & 0.67 \\
\hline
\end{tabular}

Values are presented as $\log _{2}$ values. miRNA or miR, microRNA; n.d., not detected.

OL mice, and relative miRNAs expression levels (MLO-Y4 exosomes/ST2 exosomes and MLO-Y4 exosomes/MLO-Y4 cells) are shown in Tables III and IV, respectively. With the exception of miR-208a-5p, a total of 12 miRNAs downregulated $\left(\log _{2}\right.$ value $\left.<-1\right)$ in the OL mouse plasma were expressed at higher levels in the MLO-Y4 exosomes compared with the levels in ST2 exosomes (Table III). By contrast, 10 upregulated $\left(\log _{2}\right.$ value $>2$ ) enriched miRNAs were undetectable in both exosomal and cellular fractions of MLO-Y4 cells with the exception of miR-92a-3p (Table IV). These results suggest that these exosomal miRNAs downregulated in OL mouse plasma may be derived from exosomes secreted by osteocytes.

Small RNA deep sequencing of exosomes derived from MLO-Y4 cells. The microarray method, relying on sequence hybridization to appropriately-designed annealing probes, can only detect annotated miRNAs, as well as immature
miRNAs, such as pre- or pri-miRNAs, which include the same sequences that would be detected in mature miRNAs. Sequencing-based technology reveals the entire repertoire of expressed small RNAs (23). In order to analyze the abundance of miRNAs and to identify the miRNA sequences that detect only mature miRNAs from the MLO-Y4 exosomes, a small RNA cDNA library was generated and deep sequencing was performed. A total of 20,000,000 raw reads were obtained from the sample. Subsequent to removing adapter sequences and selecting by size differences (1-17, 18-25 and >26 nt), $9,954,039$ clean reads with sizes within the ranges of $1-17$, 18-25 and $>26$ nt were generated (Fig. 1). In the 18-25 nt size range of small RNA sequences, small RNA characterization analysis focused on searching release 21 of miRBase. Fig. 1 demonstrates a summary of the different types of small RNAs detected in the samples, according to current miRNA annotations. Of the total reads, 195,581 were annotated to mouse 
Table V. Summary of miRNA read counts from exosomes of MLO-Y4 cells.

\begin{tabular}{|c|c|}
\hline No. of read counts & miRNA \\
\hline \multirow[t]{5}{*}{$30-49$} & $\operatorname{miR}-3473 b$ \\
\hline & miR-6240 \\
\hline & miR-145a-5p \\
\hline & miR-1195 \\
\hline & miR-6538 \\
\hline \multirow[t]{7}{*}{$50-99$} & miR-690 \\
\hline & $\operatorname{miR}-5121$ \\
\hline & miR-24-3p \\
\hline & miR-709 \\
\hline & miR-125a-3p \\
\hline & miR-3102-5p \\
\hline & miR-20a-5p \\
\hline \multirow[t]{20}{*}{ Up to 100} & miR-23b-3p \\
\hline & miR-130a-3p \\
\hline & miR-140-3p \\
\hline & $\operatorname{miR}-23 a-3 p$ \\
\hline & $\operatorname{miR}-29 a-3 p$ \\
\hline & miR-214-3p \\
\hline & miR-3473e \\
\hline & miR-125b-5p \\
\hline & miR-16-5p \\
\hline & miR-22-3p \\
\hline & $\operatorname{miR}-27 a-3 p$ \\
\hline & $\operatorname{miR}-92 a-3 p$ \\
\hline & miR-17-5p \\
\hline & $\operatorname{miR}-221-3 p$ \\
\hline & miR-484 \\
\hline & miR-671-5p \\
\hline & miR-125b-1-3p \\
\hline & miR-191-5p \\
\hline & miR-451a \\
\hline & miR-615-5p \\
\hline
\end{tabular}

miRNA or miR, microRNA.

miRNA sequences (Fig. 1). In addition, 527 known mouse mature miRNAs were detected, as well as 400 stem-loop structures of full-length and truncated pre-miRNAs (Fig. 1). Among these detected miRNAs, 32 miRNAs were found that were enriched in exosomes derived from MLO-Y4 cells, as shown in Table II, and the read counts were up to 30 (Table V). Furthermore, among these 32 miRNAs, miR3473b and miR3473e were reduced in exosomes from OL mouse plasma (Table VI). These results indicated that MLO-Y4 exosomes contain varying expression levels of characterized miRNAs.

\section{Discussion}

To the best of our knowledge, the present study is the first to describe the effect of the lack of osteocytes on the miRNA
Table VI. Summary of read counts $(>30)$ of upregulated or downregulated miRNAs in osteocyte-less mouse plasma exosomes.

\begin{tabular}{llrc}
\hline Regulation & miR ID & $\begin{array}{c}\text { Read } \\
\text { counts }\end{array}$ & $\begin{array}{c}\text { Relative miRNA } \\
\text { expression }\end{array}$ \\
\hline Upregulated & miR-29a-3p & 832 & 1.51 \\
& miR-16-5p & 1050 & 1.58 \\
& miR-92a-3p & 13672 & 2.08 \\
& miR-221-3p & 5998 & 1.77 \\
& miR-484 & 1745 & 1.59 \\
& miR-451a & 385 & 1.71 \\
Downregulated & miR-3473b & 48 & -1.52 \\
& miR-3473e & 116 & -1.51 \\
\hline
\end{tabular}

${ }^{\mathrm{a}}$ Expression of OL plasma exosome/WT plasma exosome, with values presented as $\log _{2}$ values. miRNA or miR, microRNA.

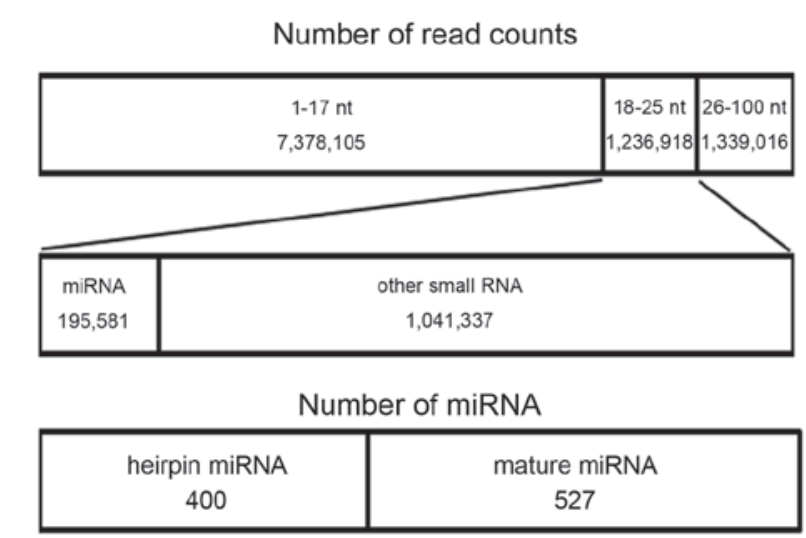

Figure 1. Overview of RNA profile, read counts of RNAs and number of miRNAs from MLO-Y4 exosomes. miRNA, microRNA.

levels of circulating plasma exosomes. There are several possible explanations for the alteration of miRNA expression levels in circulating exosomes in the blood of OL mice: i) Low production of osteocyte-derived exosomes influences the miRNA expression level of circulating exosomes in the OL mice; ii) damaged osteocytes secrete numerous apoptotic bodies or other microparticles, which then influence the miRNA expression level of circulating exosomes in the OL mice; and iii) there is no change in the level of the secretion of exosomes and/or microvesicles, apoptotic bodies and other microparticles, and only alteration of the miRNA expression level in exosomes occurs from bone and other tissues.

In the present study, miRNA microarray analysis identified 12 miRNAs (miR-3473a, miR-3473b, miR-3473e, miR-5128, miR-6244, miR-6239, miR-5132-5p, miR-705, miR-208a-5p miR-3104-5p, miR-1224-5p and miR-5621-5p) that were downregulated $\left(\log _{2}\right.$ value $\left.<1.0\right)$ in OL mouse plasma exosomes compared with WT plasma exosomes. These 12 miRNAs were expressed at higher levels in MLO-Y4 exosomes than in ST2 exosomes, with the exception of miR-208a-5p. A possible explanation of these findings could be that the decrease of 
miRNAs in plasma exosomes of OL mice is caused by a decrease in leakage or secretion of exosomes from osteocytes, as described earlier in the first hypothesis, suggesting that osteocytes secrete exosomes which then circulate in the blood.

Since osteocytes release apoptotic bodies during apoptosis (24), it is possible that damaged osteocytes may secrete a large number of exosomes. However, this second possibility may be excluded since the top 10 upregulated miRNAs (miR-122-5p, miR-133b-3p, miR-133a-3p, miR-25-3p, miR-92b-3p, miR-19a-3p, miR-92a-3p, miR-144-3p, miR-15b-5p and miR-29c-3p) were not detected in the exosomal or cellular fraction of MLO-Y4 cells, with the exception of miR-92a-3p. In addition, among the top 10 downregulated miRNAs, miR-3473b and miR-3473e were also detected in MLO-Y4 exosomes by small RNA deep sequencing. Previously, Sato et al $(5,20)$ demonstrated that ablation of osteocytes in mice (OL mice) leads to severe lymphopenia, due to the lack of a lymphoid-supporting stroma in the bone marrow and thymus, and to the complete loss of white adipose tissues. We cannot exclude the possibility that these miRNAs are secreted by cells of other organs or tissues as a result of the ablation of osteocytes. In fact, downregulation of miR-3473 has been reported as one of the miRNAs that may involve brain dysfunction following acute liver failure (25). Consequently, certain upregulated miRNAs (miR-29, miR-484 and miR-221) are reported to be involved in energy metabolism through the regulation of liver function, glucose response of $\beta$-cells, adipogenesis and obesity (26-28).

In the present study, cultured MLO-Y4 cells were found to release exosomes. Histologically, it has been reported that, during apoptosis, osteocytes release protein-containing $20 \mathrm{~nm}$ to $1 \mu \mathrm{m}$ microvesicles known as osteocyte apoptotic bodies, which stimulate osteoclastogenesis (23). The results of confocal laser scanning microscopy analysis reveal that osteocytic processes extend to the vascular-facing surface of osteoblasts in vivo (29). This suggests that osteocyte-derived exosomes may be released not only into the local bone microenvironment, but also into nearby blood vessels. Exosomes contain several groups of proteins, lipids and genetic material, including miRNAs (6). The deep sequencing results of the present study revealed that MLO-Y4 exosomes contain both mature miRNAs and hairpin pre-miRNAs. Although the role of pre-miRNAs in exosomes remains unclear, previous studies also showed that exosomes contain predominantly pre-miRNAs (30-32). The current study suggests the possibility that osteocyte-produced exosomes circulate throughout the body, and then transfer their component signaling molecules, including miRNA and pre-miRNA, by binding to a cell surface, followed by fusion and internalization by recipient cells in other organs and/or tissues, as previously described (33).

In conclusion, the current study has demonstrated that ablation of osteocytes in mice alters the miRNA levels of plasma exosomes, and that MLO-Y4 cells secrete exosomes containing miRNAs. In addition, the present findings suggest that osteocytes secrete exosomes which then circulate in the blood. This is the first study linking osteocyte exosomes to circulating exosomes. As such, these findings may provide important new information pertaining to the molecular basis of the regulation of remote organs by bone-derived osteocytes.

\section{Acknowledgements}

The present study was supported in part by the JSPS Grants-in-Aid for Scientific Research [grant no. 15K20830 (SM)].

\section{References}

1. Bonewald LF: The amazing osteocyte. J Bone Miner Res 26: 229-238, 2011.

2. Schaffler MB, Cheung WY, Majeska R and Kennedy O: Osteocytes: Master orchestrators of bone. Calcif Tissue Int 94: 5-24, 2014.

3. Dallas SL, Prideaux M and Bonewald LF: The osteocyte: An endocrine cell ... and more. Endocr Rev 34: 658-690, 2013.

4. Quarles LD: Skeletal secretion of FGF-23 regulates phosphate and vitamin D metabolism. Nat Rev Endocrinol 8: 276-286, 2012.

5. Sato M, Asada N, Kawano Y, Wakahashi K, Minagawa K, Kawano H, Sada A, Ikeda K, Matsui T and Katayama Y: Osteocytes regulate primary lymphoid organs and fat metabolism. Cell Metab 18: 749-758, 2013.

6. Colombo M, Raposo G and Théry C: Biogenesis, secretion, and intercellular interactions of exosomes and other extracellular vesicles. Annu Rev Cell Dev Biol 30: 255-289, 2014.

7. Vlassov AV, Magdaleno S, Setterquist R and Conrad R: Exosomes: Current knowledge of their composition, biological functions, and diagnostic and therapeutic potentials. Biochim Biophys Acta 1820: 940-948, 2012.

8. Salido-Guadarrama I, Romero-Cordoba S, Peralta-Zaragoza O, Hidalgo-Miranda A and Rodríguez-Dorantes M: MicroRNAs transported by exosomes in body fluids as mediators of intercellular communication in cancer. Onco Targets Ther 7: 1327-1338, 2014

9. Simons M and Raposo G: Exosomes - vesicular carriers for intercellular communication. Curr Opin Cell Biol 21: 575-581, 2009.

10. Stoorvogel W: Functional transfer of microRNA by exosomes. Blood 119: 646-648, 2012.

11. Williams AE: Functional aspects of animal microRNAs. Cell Mol Life Sci 65: 545-562, 2008.

12. Sayed D and Abdellatif M: MicroRNAs in development and disease. Physiol Rev 91: 827-887, 2011.

13. Bushati N and Cohen SM: microRNA functions. Annu Rev Cell Dev Biol 23: 175-205, 2007.

14. Huntzinger E and Izaurralde E: Gene silencing by microRNAs: Contributions of translational repression and mRNA decay. Nat Rev Genet 12: 99-110, 2011.

15. Montecalvo A, Larregina AT, Shufesky WJ, Stolz DB, Sullivan ML, Karlsson JM, Baty CJ, Gibson GA, Erdos G, Wang Z, et al: Mechanism of transfer of functional microRNAs between mouse dendritic cells via exosomes. Blood 119: 756-766, 2012.

16. van Balkom BW, de Jong OG, Smits M, Brummelman J, den Ouden K, de Bree PM, van Eijndhoven MA, Pegtel DM, Stoorvogel W, Würdinger T and Verhaar MC: Endothelial cells require miR-214 to secrete exosomes that suppress senescence and induce angiogenesis in human and mouse endothelial cells. Blood 121: 3997-4006, S1-S15, 2013.

17. Tatsumi S, Ishii K, Amizuka N, Li M, Kobayashi T, Kohno K, Ito M, Takeshita S and Ikeda K: Targeted ablation of osteocytes induces osteoporosis with defective mechanotransduction. Cell Metab 5: 464-475, 2007.

18. Kato Y, Windle JJ, Koop BA, Mundy GR and Bonewald LF: Establishment of an osteocyte-like cell line, MLO-Y4. J Bone Miner Res 12: 2014-2023, 1997.

19. Tamura M, Sato MM and Nashimoto M: Regulation of CXCL12 expression by canonical Wnt signaling in bone marrow stromal cells. Int J Biochem Cell Biol 43: 760-767, 2011.

20. Sato M and Katayama Y: Osteocytes and Homeostasis of Remote Organs: Bone-Buried Osteocytes Talk to Remote Organs. Curr Osteoporos Rep 13: 193-197, 2015.

21. Ogawa M, Nishikawa S, Ikuta K, Yamamura F, Naito M, Takahashi K and Nishikawa S: B cell ontogeny in murine embryo studied by a culture system with the monolayer of a stromal cell clone, ST2: B cell progenitor develops first in the embryonal body rather than in the yolk sac. EMBO J 7: 1337-1343, 1988. 
22. Otsuka E, Yamaguchi A, Hirose $\mathrm{S}$ and Hagiwara $\mathrm{H}$ : Characterization of osteoblastic differentiation of stromal cell line ST2 that is induced by ascorbic acid. Am J Physiol 277: C132-C138, 1999

23. Liu CG, Calin GA, Meloon B, Gamliel N, Sevignani C, Ferracin M, Dumitru CD, Shimizu M, Zupo S, Dono M, et al: An oligonucleotide microchip for genome-wide microRNA profiling in human and mouse tissues. Proc Natl Acad Sci USA 101: 9740-9744, 2004.

24. Kogianni G, Mann V and Noble BS: Apoptotic bodies convey activity capable of initiating osteoclastogenesis and localized bone destruction. J Bone Miner Res 23: 915-927, 2008.

25. Vemuganti R, Silva VR, Mehta SL and Hazell AS: Acute liver failure-induced hepatic encephalopathy s associated with changes in microRNA expression rofiles in cerebral cortex of the mouse [corrected]. Metab Brain Dis 29: 891-899, 2014.

26. Tang X, Muniappan L, Tang G and Ozcan S: Identification of glucose-regulated miRNAs from pancreatic \{beta\} cells reveals a role for miR-30d in insulin transcription. RNA 15: 287-293, 2009.

27. Xie H, Lim B and Lodish HF: MicroRNAs induced during adipogenesis that accelerate fat cell development are downregulated in obesity. Diabetes 58: 1050-1057, 2009.
28. Williams MD and Mitchell GM: MicroRNAs in insulin resistance and obesity. Exp Diabetes Res 2012: 484696, 2012

29. Kamioka H, Honjo T and Takano-Yamamoto T: A three-dimensional distribution of osteocyte processes revealed by the combination of confocal laser scanning microscopy and differential interference contrast microscopy. Bone 28: 145-149, 2001.

30. Chen TS and Lim SK: Measurement of precursor miRNA in exosomes from human ESC-derived mesenchymal stem cells. Methods Mol Biol 1024: 69-86, 2013.

31. Melo SA, Sugimoto H, O'Connell JT, Kato N, Villanueva A, Vidal A, Qiu L, Vitkin E, Perelman LT, Melo CA, et al: Cancer exosomes perform cell-independent microRNA biogenesis and promote tumorigenesis. Cancer Cell 26: 707-721, 2014.

32. Lee HK, Finniss S, Cazacu S, Xiang C and Brodie C: Mesenchymal stem cells deliver exogenous miRNAs to neural cells and induce their differentiation and glutamate transporter expression. Stem Cells Dev 23: 2851-2861, 2014.

33. Mittelbrunn M and Sánchez-Madrid F: Intercellular communication: Diverse structures for exchange of genetic information. Nat Rev Mol Cell Biol 13: 328-335, 2012. 